\title{
Speculative Requirements: Design Fiction and RE
}

\author{
Andy Darby*, Emmanuel Tsekleves*, Pete Sawyer ${ }^{\dagger}$ \\ ${ }^{*}$ Lancaster University, $U K$ \\ ${ }^{\dagger}$ Aston University, UK \\ \{a.darby,e.tsekleves\}@lancaster.ac.uk, \{p.sawyer\}@aston.ac.uk
}

\begin{abstract}
Many innovative software products are conceived, developed and deployed without any conventional attempt to elicit stakeholder requirements. Rather, they are the result of the vision and intuition of a small number of creative individuals, facilitated by the emergence of a new technology. In this paper we consider how the conditions that enable new products' emergence might be better anticipated, making innovations a little less reliant on individual vision and a little more informed by stakeholder need. This is particularly important where a new technology would have the potential for social impact, good or bad. Speculative design seeks to explore this landscape. We describe a case study using a variant called design fiction to explore how plausible new technologies might impact on dementia care.
\end{abstract}

Index Terms - requirements elicitation, design fiction, speculative design, design thinking, creativity

\section{Introduction}

The emergence of a new technology, or the discovery of a novel way to combine existing technologies, provides opportunities to those with the vision and resources to exploit it, often in a highly disruptive way. What quantum computing will mean for existing cryptosystems is a rare example where attention is being given to the potential for disruption while the technology is still developing. However, the effects of less obviously disruptive emerging technologies seldom attract much interest until they become impossible to ignore.

Many new products, disruptive or not, are conceived, developed and deployed without any conventional exercise of requirements engineering practice. There are many good reasons for this, not least the difficulty of communicating the potential of an emerging technology and its effects to stakeholders. However, there is a case to be made for paying more attention to trying to identify the impacts of a new technology before it becomes fully realised. The question is whether, if we could find the means to explore the impact of an emerging technology, the consequences might be anticipated and controls could be discussed and planned.

In this paper we consider how the conditions that enable new products' emergence, might be better anticipated, making innovations a little less reliant on individual vision and a little more informed by stakeholder need. This is particularly important where a new or just-over-the-horizon technology would have the potential for social impact, good or bad. To do this, we have chosen to look beyond conventional RE at speculative design, which describes a basket of techniques that seek to explore this landscape. We focus particularly on one of these, design fiction, and describe a case study, the What If? project, where it was used to explore how plausible new technologies might impact on dementia care. We describe the essential features of design fiction and how it relates to $\mathrm{RE}$.

The ultimate contribution of the work described in this paper will be an understanding of if and how principles can be leveraged from speculative design to improve RE's ability to reduce the shock or negative effects that result from disruptive new technologies. At the current stage of the work we have been able to identify some general parallels with current RE practice, and identify some areas of divergence between speculative design and RE.

The rest of this paper is structured as follows. In the next section we review related work, drawing together research from RE and design thinking. Next, we introduce the 'What if?' case study that we draw upon and describe the design fiction process that we followed and the design artefacts that resulted. This is followed by a discussion of what we have discovered so far and the paper's conclusion.

\section{Related work}

In [1], "over-the-horizon" knowledge, that is knowledge involving "unknown unknowns" [2], is identified as the principle challenge to knowledge elicitation for RE. Such knowledge isn't documented anywhere, it may not be recognised by the stakeholders even if it is surfaced and its very existence may be uncertain. Yet acquiring this knowledge may be critical to the solution. Thus, for example, an as yet unrealised new technology will have an effect on the world that is uncertain. Attempting to clarify its effect is difficult because stakeholders will have difficulty envisioning the technology and the context of its use. As a result, the eventual deployment of the technology may fail, or it may achieve its goals but have unintended consequences for its users or to others. For example, at the time of writing, unintended consequences in the form of criminal funding and energy consumption can be observed arising from Blockchain. 
The effective exploitation of a new technology therefore presents a wicked design problem; one that is illformulated and where the available information is incomplete, confusing and contradictory. With the seemingly unstoppable tide of new technologies, a research community has evolved around speculative design, which allows the designer to think about the future prospectively and critically [4]. Speculative design is an umbrella term for a collection of approaches to design thinking [9] that, by paying careful attention to social context, allow the designer to explore plausible future worlds. The key idea is that by "exploring ideas before they become products or even technologies, designers can look into the possible consequences of technological applications before they happen" [3].

Because speculative design tends to focus on as yet unrealised technologies, implementation is generally infeasible. Rather, speculative design provides insights into the kinds of products or product features that might become feasible and their value to potential stakeholders, the barriers to adoption a technology might face, and wider implications for society through public policy, regulations, ethical frameworks or law. Speculative design is thus more about requirements, goals and environmental context than it is about design in the way that software engineers think about design.

Conventional RE is about identifying the design space of the digital product; a design space that is bounded by a known or knowable set of cultural, ethical, legislative, etc. constraints that need to be understood but go largely unquestioned because they are out of scope. With speculative design, by contrast, these constraints are within scope and may even become the focus of subsequent (re-)design. When approached from an affirmative [3] perspective, speculative design encourages the explicit questioning of constraints on the design space and testing their fitness-for-purpose in the envisioned future world.

While Speculative design includes techniques familiar to $\mathrm{RE}$, such as brainstorming and rapid prototyping, design fiction supplements these with techniques borrowed from art, literature, film, psychology, philosophy, anthropology and ecology. Its aim is "creative provocation, raising questions, innovation, and exploration" [5]. One of its fundamental principles is the negation of the status quo and initiation of an exploration of possible worlds through confrontation with tangible objects or processes; diegetic prototypes. Diegetic prototypes are realisations of future technologies that "demonstrate a technology's need, viability and benevolence" [6] with sufficient fidelity to "suspend disbelief about change" [4].

Design fictions are explorations of particular solution spaces made possible by combining current and emergent technological advances with society's slow-changing social, legal and ethical practices. To that end, various forms of speculative design, including codesigned design fiction [7], have been tested with government to assess their potential to contribute to real-world policy development [8].

\section{The 'What If?' case study}

Significant resources are being invested in developing new assistive and diagnostic technologies for the elderly or those living with dementia (e.g. [10], [11]) as increasing life expectancy leads to increases in the incidence of dementia. The potential health-related benefits offered by technology are counterweighted by societal, legal and ethical challenges [14] and many governments are seeking to define forwardlooking policies on dementia care (e.g. in the UK [12]).

As emergent technologies and government policies mix and shape our futures, there is therefore a need to investigate potential consequences for people living with dementia, their carers, families and for society at large. A key part of this is the need for public engagement and debate between policy makers and citizens.

This need has emerged at a time when there is a trend to include more collaborative methods of engaging with the public, alongside traditional consultative practices, to encourage comment and debate on government policy [13]. This has included experimentation with various forms of speculative design being used to address policy [8].

This was the background to What If? project; a collaboration between design thinking and requirements engineering researchers. The overall goal of our research is to improve our ability to anticipate the effects of new technologies and in particular the societal impacts they might have. To be effective, we need to be able to do this in a systematic way so a further, more specific goal is to perform a design speculation to gain insights into the extent to which existing RE practice can be applied, where RE currently lacks the techniques to investigate the future and how it can be augmented to fill the gaps.

As a first step in achieving these goals, the research question the What If? case study is designed to answer is: How can stakeholders, domain experts and policy makers involved in dementia care be provoked to think about the impact of new technologies?. To answer this we address the following sub-questions:

1) Is participative design fiction effective in stimulating this thinking?

2) What are the crossovers between design fiction and $\mathrm{RE}$ for speculating about the technological future?

\subsection{Methodology}

Participative design fiction is the probe into the near future that we have selected. We are using envisioned technology as a prop to uncover the issues that might eventually serve as requirements or constraints on technology products emerging in this space, and on the policy that will form part of the technology's wider environment.

In outline the project was planned as follows:

1) Run a set of workshops to develop plausible design concepts to represent envisioned technologies generated in response to participant-identified issues, 
2) Select a design concept and develop it into a diegetic prototype,

3) Develop the narrative for the design fiction; in our case a film in which actors employ the diegetic prototype in a realistic dementia care scenario in a plausible envisioned future,

4) Use the film to collect feedback on the potential future from the workshop participants,

5) Refine the diegetic prototype and film scenario based on the feedback, and

6) Disseminate the (new) film to stimulate debate about dementia policy.

7) Synthesise a set of effective principles in the application of design fiction and speculative design to reasoning about the technological near future, with the ultimate aim of operationalising them (we hope) as an augmentation to the requirements engineer's toolkit.

At the time of writing, we are at stage 5 .

\subsection{The workshops}

Five workshops were planned, focused on two groups of stakeholders: healthy older adults (age range 60-90); and professionals from Age UK, a charity championing elderly people in the UK and a key stakeholder in government dementia policy. Four workshops were planned for the older adults. Of these, two didn't recruit sufficient participants and one failed to yield results because of cultural obstacles within the community it targeted.

For the remaining two workshops, participants began by discussing their understanding of how technology has shaped their lives. Next, they shared their experience of dementia through a series of question cards. We then introduced the Prime Minister's Dementia Challenge [15] (a document that formed the background to [12]) to participants for discussion and critique. Participants were invited to consider what they found reassuring, what was worrying, what was missing from the document, what assumptions underpinned the document and who the beneficiaries of the policy were. These insights were then prioritised and assessed as to their positive or negative impacts.

Participants were asked to consider current technological interventions into dementia-related areas before they were introduced to 10 technology trends [16]: the device mesh; ambient user experience; 3D-printing materials; information of everything; advanced machine learning; autonomous agents and things; adaptive security architecture; advanced system architecture; mesh app and service architecture; and internet of things architecture and platforms.

In groups, participants were invited to select one of the prioritised issues that arose from their consideration of the Prime Minister's challenge, and informed by the technology trends, begin to develop outlines (design speculations) of a technology-based way to address the issue (Figure 1). To help stimulate creativity the SCAMPER cognitive heuristic was used to challenge the participants' design concepts, with

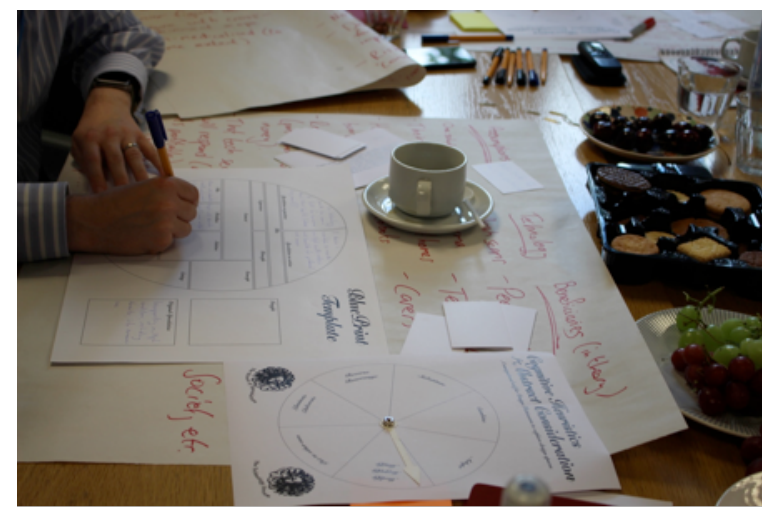

Figure 1. Participants working on their design concepts

chance abstract concept selections being used to test and question the participants' original concepts. This encouraged participants to explore the elements of a product story and gave them space to form and reform their understanding of their original speculation. The final activity took the design concepts generated and classified them as to their positive or negative impact and their various probabilities, plausibilities, or possibilities as they extended out into the future.

Consideration of [15] identified two areas of policy that the participants believed were not or under-represented. Of these, the one that presented the greatest challenge and which we therefore selected to be developed further, was support for people with multiple morbidities (or comorbidities where people have one or more chronic conditions in addition to their dementia [17]).

The participant group that developed the design concept stated "People with dementia typically have multiple conditions, which prevent effective management of their overall health." They envisaged a device, called the Multi-monitor that tracked vital health metrics to assist clinicians when a person with dementia was admitted to hospital and to assist day-to-day health management.

The Multi-monitor concept drew on plausible improvements in the reliability of health sensors and associated metrics, and in natural language processing. It was conceived to explore the questions "Can technology provide the means to talk for someone about their health (where they cannot)?" and is it feasible to have "a device [that] is available to monitor multiple health metrics and translate for professionals and carers?"

\subsection{The Multi-monitor diegetic prototype}

Diegetic prototyping uses as its starting point a design concept envisioning a possible future world. The designer seeks to realise the design concept to a degree of fidelity sufficient to articulate the speculation to an audience of stakeholders. The diegetic prototype is then 'socialised' within the possible future world through its interactions with others in the design fiction through storyboarding, improvising and scripting. The diegetic prototype need not 


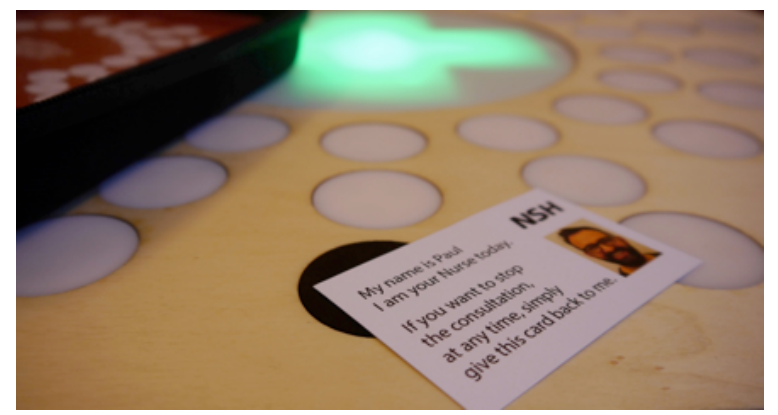

Figure 2. Healthcare professional's authorisation card

be fully functional, but it does need key qualities to be sufficiently developed to appear convincing in the chosen medium of narrative fiction.

The Multi-monitor concept was envisioned as a single artefact but to help better explicate the concept a rich set of artefacts were used to flesh out, and lend credence to the design fiction. Collectively, these artefacts were named the Mentian consultation system.

The principal Mentian artefact is the consultation system implemented as a computer integrated within a table around which a consultation would take place. The table integrates sensor data, responding verbally to queries and presenting information to healthcare professionals and carers. The table form was chosen as a convenient way to house the hardware and as a tangible way of keeping the data close to the person with dementia, as it would be sited in their own home.

On the tabletop, a circular pattern featuring several white circles is used to visualise the idea of multi-morbidities, with each white circle representing a health sensor. The table is constructed of laser cut plywood and houses an Arduino Uno, an NFC reader, colour LED lights and battery packs.

A healthcare professional's identity card doubles as a digital authorisation card, initiating the system when presented to the tabletop, and providing a mechanism for the removal of patient consent to the consultation. On the front side, alongside a picture of the nurse and a health service logo, the text reads:

My name is Paul I am your Nurse today. If you want to stop the consultation, at any time, simply give this card back to me.

On the rear side is a Near Field Communication tag. Consent for the consultation hinges on the authorisation card being laid on the black health service logo on the table. When the authorisation card is placed on the logo the central circle of the table lights up with a green cross and the system is ready for use (see Figure 2).

In Figure 3, the Mentian consultation table is in the foreground. Visible on the tabletop is a case containing a set of Mentian sensor panels. A single panel provides sensors tracking a particular co-morbidity, for example $H D$ indicating Heart Disease; UTI indicating Urinary Tract Infection; and $C I$ indicating Chest Infection. The healthcare professional can select the panel that represents the particular comorbidity that they want to interrogate the Mentian system

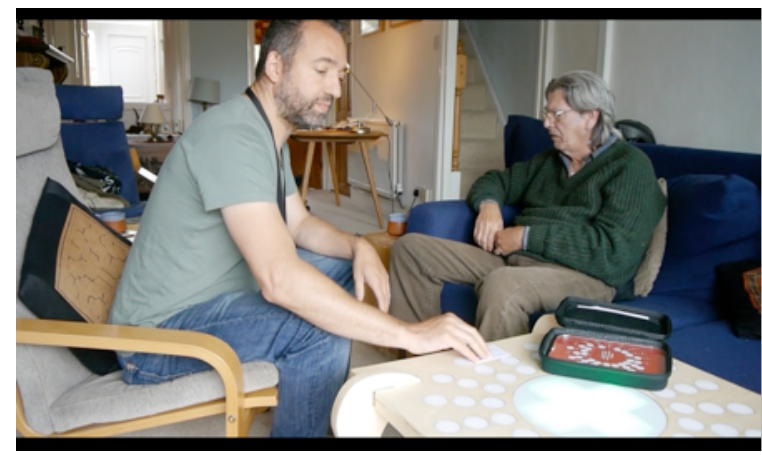

Figure 3. Still from the design fiction film

about, uploading the sensor data to the Mentian system when selected. The selection of a panel provides a strong visual cue to as to which condition is the focus of the consultation at any particular point in time.

Once uploaded from the sensors, the data resides in the Mentian consulting system. Thus the personal health data stays in the dementia sufferer's home, as if it were another of their everyday belongings.

\subsection{The design fiction}

The audience for the design fiction comprises stakeholders whose insights would be essential for anticipating the near future represented by the design fiction. For Mentian, the intended audience is a group of professionals convened by Age UK with a deep understanding of dementia care. The design fiction film was accordingly developed around a scenario that featured a person living with dementia in his own home in consultation with a health professional making use of Mentian.

In the design fiction film, the lead author took the role of the Nurse Practitioner, Paul, and professional actors took the role of Ron Murdoch, a person with mid-stage dementia, and Mentian.

The design fiction was improvised over the course of a morning. Over a number of improvisations, the group devised and shaped the consultation and the various roles within it and focussed their interactions with the props. The lead author carried out the filming and edited the material for presentation of a work-in-progress version; the first version of the design fiction. Figure 3 shows a still from the film.

To collect feedback and refine the design fiction before exposure to our target audience, the film was shown to our Age UK liaison and participants from the workshop that had conceived the multi-morbidity design concept.

\section{Findings and discussion}

To date, the early feedback we have received from the workshop participants and age UK liaison falls into three categories: insights into dementia policy; insights into the technology envisioned by Mentian; and insights into the design fiction process that we used. 


\subsection{Dementia policy insights}

First, the initial workshops were successful in identifying incompleteness in the policy document [15] including the absence of consideration of multiple morbidities. These are already well known problems with dementia care [18], [19]. However, it is the intersection of technology and policy that is the real focus of our work, and this is discussed below.

\subsection{Technology insights}

We are unaware of any research into technology for managing multiple morbidities in dementia patients, so stimulating an investigation into the possibility of such a technology is itself a contribution to dementia care.

The use of Gartner's 10 technology trends [16] as a lens through which to view the multiple morbidities problem, stimulate a design concept and then challenge this using SCAMPER was, we believe, novel when applied to dementia policy and led directly to the multi-monitor design concept.

The envisioned technology represented by the Mentian design fiction stimulated doubts about reliance on adhesive sensors. Stick-on sensors can irritate sensitive skin, they can get washed or picked off and they won't work for a urinary tract infection (for example).

The sensors stimulated a debate about inserting sensors under the skin. The idea is interesting but raises some awkward ethical questions about consent in people with cognitive impairment. These haven't been resolved yet, but the fact that they have been surfaced anticipates issues that may become urgent in the near future, with implications for medical technologies, ethics and care policy.

Other design features have not yet attracted comment. We think that the consultation scenario might have made the film inaccessible to participants without experience of consultations. However, it did have the merit of stimulating debate and we hope this will continue as we expose the design fiction to our expert panel.

\subsection{The design fiction process}

The workshop participants who developed the Multimonitor design concept questioned how much of their input had found its way into the Mentian system. The hand-over from workshop participants developing design concepts to the designers developing a fully-realised design fiction appears to lose traceability between goals and design features. This may have been exacerbated by the several months it took to design and build the diegetic prototype and the design fiction and to produce and edit the film.

There may be two responses to this problem. The first is rooted in modern design thinking and involves moving from the co-design practices in our design fiction process to a truer embodiment of co-creation. Here, development of the diegetic prototype becomes a longitudinal collaboration between the designers and the workshop participants. This would of course require much more of a time commitment from the workshop participants and this could be problematic (e.g.) for the Age UK professionals. The second solution may be to make the process more agile and expose the prototype and design fiction to the workshop participants more frequently and in smaller increments.

\section{What next?}

With reference to the process set out above, we are revising the Mentian diegetic prototype and design fiction in the light of the initial feedback (step 5). When this is complete, we will present the film to the panel of experts convened by Age UK to elicit feedback through a structured workshop (step 6). The purpose of this evaluation is not to validate Mentian. Its value will lie in the extent to which Mentian serves as a catalyst for discussion, helping the panel to provide insights to the multi-monitor idea that would point to areas where policy needs to be developed (e.g. around ethics of subcutaneous sensors) and perhaps even some generic goals identified for technology for managing multiple morbidities in people with impaired cognition (e.g. around the utility of natural language or the visualisation of co-morbidities).

The final step (step 7) will be to generalise away from Mentian and What If? towards ultimately defining a repeatable process that can be applied to different near-future contexts. We already have some preliminary insights into the efficacy of participative design fiction, where existing $\mathrm{RE}$ principles are crucial (especially traceability) and also the barriers to its use. These will be added to and concretised as the work draws to its conclusion.

\section{Implications for RE}

What have we learned that is of relevance to RE?

- Speculating about the future. RE's reach does not extend very far into the future. RE is usable for green-field problems, but (a) there are few incentives to invest in reasoning about the future, and (b) RE lacks the tools to engage stakeholders in reasoning about the future. In terms of (a), technologies sometimes emerge that have significant societal implications and it may repay indulging in more speculative analyses about these to better prepare for their impact. In terms of (b), the What If? project provides some evidence that a participatory approach to design fiction facilitates these speculations in a way that can engage tomorrow's stakeholders.

- Traceability still matters. To retain stakeholders' engagement and sense of problem ownership, they want to see how their upstream ideas are manifested in the more developed future world downstream; the diegetic prototype and design fiction. Further, the ability to trace back upstream to the origin of the requirements to test them against reality once the technology is realised will be critical to the requirements' acceptance. 
- It's all about creativity. Good RE has always depended upon creative practitioners [20]. Speculative design requires tools to stimulate creativity in stakeholders who may not consider themselves to be overtly creative in the sense that a professional designer (or requirements engineer) is. We used SCAMPER to challenge and expand the workshop participants' design concepts. Goal modeling may have a role to play too, since it explicitly supports reasoning about alternative solutions and the tradeoffs they entail.

- It isn't cheap or quick. To maintain a credible simulacrum of the future the fidelity of the diegetic prototype (or at least some of its key qualities) has to be high and it must be suited to the medium selected for the design fiction. Moreover, the contextualisation of the diegetic prototype provided by the design fiction also needs careful selection according to the stakeholder participants experience. All this implies a somewhat protracted process that may be at odds with project sponsors' and stakeholder participants' expectations.

\section{Conclusions}

We are increasingly exposed to new technologies with the potential for societal impact, so we should take steps to anticipate what the effects of at least some of these technologies might be. We believe that this lies within the remit of RE, but that RE is ill-equipped to support it. We are investigating the use of participatory approaches to design fiction for developing plausible future worlds and simulating the place of just-over-the-horizon technologies within them.

The paper reports work in progress in the use of design fiction to explore a plausible near future world in which the care of people suffering from dementia and one or more co-morbidities is supported by technology. This technology is currently represented by the first version of a diegetic prototype that we call Mentian. Early validation of Mentian by workshop participants who created the design concept on which it is based, has shown up some important issues applying to the envisioned technology, the developing policy around dementia care and the design fiction process itself. Amongst these are the importance of traceability and the stimulation of creativity. A more extensive evaluation of Mentian by a panel of experts is planned in the next stage of the project, when we expect to be able to consolidate lessons learned and their implications for near-future RE.

\section{Acknowledgment}

This work is funded by EPSRC's Digital Economy programme (RCUK Grant EP/G037582/1), which supports the HighWire Centre for Doctoral Training at Lancaster University. (http://highwire.lancs.ac.uk)

\section{References}

[1] Sutcliffe, A., Sawyer, P.: "Requirements elicitation: Towards the unknown unknowns", proc. 21st IEEE International Requirements Engineering Conference (RE 2013), pp 92-104.

[2] Gervasi, V., Gacitua, R., Rouncefield, M., Sawyer, P., Kof, L., Ma, L., Nuseibeh, B., Piwek, P., de Roeck, A., Willis, A., Yang, H.: "Unpacking Tacit Knowledge for Requirements Engineering, in W. Maalej, A. Kumar Thurimella (Eds), Managing Requirements Knowledge, Springer, 2013, pp 23-47.

[3] Dunne, A., Raby, F.: "Speculative everything: design, fiction, and social dreaming", MIT Press, 2013.

[4] Auger, J.: "Speculative design: crafting the speculation". Digital Creativity, 24(1), 11-35, 2013.

[5] Bleecker, J.: "Design Fiction: A short essay on design, science, fact and fiction". Near Future Laboratory. 2009.

[6] Kirby, D.: "The Future is Now: Diegetic Prototypes and the Role of Popular Films in Generating Real-world Technological Development". Social Studies of Science, 40(1), 41-70, 2010.

[7] Tsekleves, E., Darby, A., Whicher, A., Swiatek, P: "Co-designing design fictions: a new approach for debating and priming future healthcare technologies and services". Archives of Design Research, 30(2), 521. 2017.

[8] Kimbell, L.: "Applying design approaches to policy making: discovering policy lab". University of Brighton. 2015.

[9] Buchanan, Richard. "Wicked Problems in Design Thinking." Design Issues, 8(2), 5-21. 1992.

[10] Sawyer, P., Sutcliffe, A. Rayson, P. and Bull, C.: "Dementia and social sustainability: challenges for software engineering", Proc. 37th IEEE Int. Conference on Software Engineering (ICSE15), 2015.

[11] Stringer, G., Couth, S., Brown, L., Montaldi, D., Gledson, A., Mellor, J., Sutcliffe, A., Sawyer, P., Keane, J., Zeng., X., Rayson, P., Leroi, I.: "Can you detect early dementia from an email? A proof of principle study of daily computer use to detect cognitive and functional decline", International Journal of Geriatric Psychiatry, 2018.

[12] Department of Health and Social Care. (2015, May). 2010 to 2015 government policy: dementia [www.gov.uk]. Retrieved from https://www.gov.uk/government/publications/2010-to-2015government-policy-dementia/2010-to-2015-government-policydementia

[13] Bradwell, P., Marr, S. "Making the most of collaboration an international survey of public service co-design" Annual Review of Policy Design, 23(53). 2008.

[14] Brown, I., Adams, A.: "The ethical challenges of ubiquitous healthcare". International Review of Information Ethics, 8(12), 5360. 2007.

[15] Department of Health. "Prime Minister's challenge on dementia", 2020.

[16] Cearley, D., Burke, B., Walker, M.: "Top 10 Strategic Technology Trends for 2016" (No. G00291818). Stamford: Gartner. 2015.

[17] Scrutton, J., Brancati, C.: "Dementia and comorbidities: Ensuring Parity of Care". London: International Longevity Centre.

[18] Stirling, C., Andrews, S., Croft, T., Vickers, J., Turner, P., Robinson, A.: "Measuring dementia carers' unmet need for services - an exploratory mixed method study", BMC Health Services Research, 10(122), 2010.

[19] Kuo, S-C, Lai, S-W., Hung, H-C., Muo, C-H., Hung, Liu, L-L., Chang, C-W., Hwu, Y-J., Chen, S-L., Sung, F-C.: "Association between comorbidities and dementia in diabetes mellitus patients: populationbased retrospective cohort study", Journal of Diabetes and Its Complications, 29(8), 1071-1076, 2015.

[20] Maiden, N., Jones, S., Karlsen, K., Neill, R., Zachos, K., Milne, A.: "Requirements Engineering as Creative Problem Solving: A Research Agenda for Idea Finding," 18th IEEE International Requirements Engineering Conference (RE'10), Sydney, NSW, pp. 57-66, 2010. 